\title{
Adaptive Frequency Domain Identification for ANC Systems Using Non-stationary Signals
}

\author{
Allahyar Montazeri \\ Engineering Department, Lancaster University, Lancaster, UK \\ Email: a.montazeri@lancaster.ac.uk \\ Saurav Karna \\ Ilmenau University of Technology, Ilmenau, Germany \\ Email: imsauravkarna@gmail.com
}

\begin{abstract}
The problem of identification of secondary path in active noise control applications is dealt with fundamentally using time-domain adaptive filters. The use of adaptive frequency domain subband identification as an alternative has some significant advantages which are overlooked in such applications. In this paper two different delayless subband adaptive algorithms for identification of an unknown secondary path in an ANC framework are utilized and compared. Despite of reduced computational complexity and increase convergence rate this approach allows us to use non-stationary audio signals as the excitation input to avoid injection of annoying white noise. For this purpose two non-stationary music and speech signals are used for identification. The performances of the algorithms are measured in terms of minimum mean square error and convergence speed. The results are also compared to the time domain NLMS algorithm for the same scenario. The proposed delayless algorithms have a closed loop structure with DFT filterbanks as the analysis filter. To eliminate the delay in the signal path two different weights transformation schemes are compared.
\end{abstract}

Index Terms - Active Noise Control (ANC), Delayless subband adaptive filter, Frequency domain adaptive filter

\section{INTRODUCTION}

Increase in the number of industrial equipment has made acoustic noise a major problem in modern societies. Although these are traditionally handled by using passive techniques like enclosures, barriers and silencers they are large, costly and ineffective at low frequencies. To overcome the shortcomings of passive techniques active noise control is proved to be a viable complement [1].

The simplest scenario for implementation of an active noise control system is the experimental duct and associated schematic shown in Fig. 1 [2]. The reference signal $u(n)$ is fed to the adaptive filter by the reference microphone. Error microphone receives the superposition of primary noise from the noise source through unknown primary plant and the secondary noise is sent through the canceling loudspeaker. The residual noise received at the error microphone is shown as $e(n)$ in Fig. 1 and used to update the weights of the adaptive filter. Based on the principle of superposition the primary noise $d(n)$ is canceled by the secondary noise of equal amplitude but opposite phase $y(n)$. In most of real active noise control systems not only the primary noise has a non-stationary nature but also the surrounding environment is time varying and prone to change $[3,4,5]$. One of the commonly used adaptive algorithms to cope these problems is the so called Filtered-x least mean square
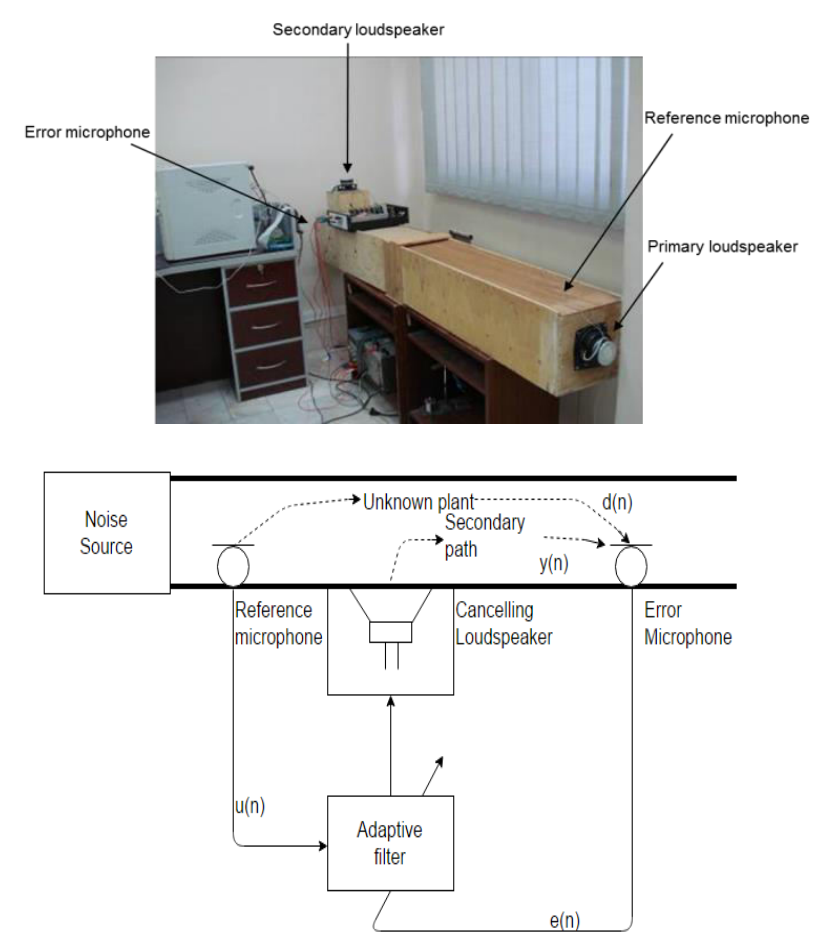

Figure 1. Single channel active noise control setup and it schematic [2].

algorithm [7]. The idea of filtered-x family of algorithms revolves around this fact that the reference signal should be filtered with an estimation of secondary path. Despite of simple and widely used filtered-x LMS algorithm in different applications analysis shows that the algorithm performs poorly in front of highly correlated and nonstationary incident noises [6]. On the one hand increase in the length of the adaptive filter results in increase in the complexity of the system, increased level of minimum mean square error, and reduction in convergence speed of the least mean square (LMS) algorithm. On the other hand, FxLMS algorithm has limited capability in tracking non-stationary signals and its stability robustness is 
subject to changes based on the accuracy of the estimated model of the secondary path $[6,7,8]$. To address the slow convergence, incapability of the algorithm in tracking non-stationary signal, and reducing the computational burden of the algorithm some variants are proposed [9, $10,11,23]$. One way to improve the robustness of algorithm with respect to the inaccuracies of the secondary path is online identification of secondary path $[12,13,14]$. In all of these approaches identification is performed in time-domain. However, a more effective method which is the focus of this paper is to identify the secondary path in the frequency domain. In this method by decomposing the signal to different subbands it is possible to generate signals whose frequency range corresponds to the passband of the analysis filters. Further downsampling of subband signals results in reduced spectral dynamic range. At the same time the length of the adaptive filters in subband techniques is shorter allowing larger step sizes to be used and again increase the convergence rate [22].

Early approaches in subband structures relied on overlapping filterbanks and critical subsampling [15]. However, it resulted in aliasing components in the output. In [16], non-overlapping filterbanks were introduced but it resulted in spectral gaps. Subband structure based on the polyphase decomposition is introduced in [17]. Filterbank structures with critical sampling of subband signals with sparse subfilters have been discussed in [18]. It results in better convergence behavior. In [19], adaptive filtering in subbands has been discussed for computational savings and better convergence rate. Adaptive cross filter between the subbands have been employed for the perfect reconstruction. Adaptive filtering at a lower decimation rate, due to subband processing, reduces the computational complexity. Also, the reduction of spectral dynamic range in each subband leads to faster convergence. However, the main anomaly of such kind of algorithm is the delay introduced in the signal path due to bandpass filters in the subband used to derive bandpass signals. The structure developed in [20], reduces the delay significantly. In this, adaptive weights are calculated in the subband and then collectively transformed into the full band filter coefficients. An additional advantage of this technique is reduction in the aliasing effects. An improvement of this structure by introducing the fractional delays in the polyphase component of the prototype filter is proposed in [21]. This eliminates the need for adaptive cross filters and hence the unknown system is modeled more accurately in a closed loop scheme.

In this paper the problem of identification of secondary path in ANC systems using delayless subband adaptive filtering is investigated. To avoid injection of auxiliary noise in ANC applications it is highly desirable to identify the secondary path using the existing music or speech signals. Due to the non-stationary nature of these signal adaptation of filter weights is challenging task. Here the performances of two adaptive subband filters for such applications in terms of speed of convergence, achievable minimum mean square error, and computational complexity are compared. The remainder of the paper is organized as follows. In section II, the structure of the delayless frequency domain adaptive system identification is introduced. Different weight transformation schemes and computational complexity of different algorithms are compared in section III. Simulation results are presented in section IV and finally concluding remarks are given in section $\mathrm{V}$.

\section{DELAYLESS FREQUENCY DOMAIN ADAPTIVE FILTERING}

The block diagram of the delayless frequency domain active sound control system is shown in the Fig. 2. The adaptive filter $W(z)$ is implemented directly in the time domain to avoid delay caused by collecting $N$ samples. The convolution is performed by multiplication in the frequency domain. It is to be noted that although full band filter weights is in the time domain all the filter updating is performed in the frequency domain. In Fig. 1 $P(z)$ in the block diagram represents the transfer function from the noise source to the error source. Convolution of the reference signal $x(n)$ with the primary path impulse response, gives the desired signal $d(n)$. The length of the primary adaptive filter is $L$. The reference signal in the secondary path is decomposed into subband signals by using polyphase FFT. A DFT filter bank, is constructed from the $K$ length prototype filter by modulation. The analysis filters of an $M$-channel DFT filter bank are obtained as:

$$
H_{i}(z)=H\left(z e^{-j 2 \pi i / M}\right), i=0,1, \ldots M-1
$$

Here, $H(z)$ is the real valued prototype low pass filter with a cutoff frequency of $\pi / \mathrm{M}$. Shifting of low pass filters to the right by the multiples of $2 \pi / \mathrm{M}$ gives the complex modulated bandpass filters. The impulse response coefficients of $H_{i}(z)$ and $H_{M-1}(z)$ are complex conjugates of each other. Therefore, for real valued signals only the first $M / 2+1$ subbands need to be processed. The pseudo error signal is also decomposed into number of subbands using the same DFT filter bank as above. Here, $e(n)$ is the residual noise from the error sensor. The weight adaptation is applied on the subfilters using the subband signals $u_{i, D}(k)$ and $e_{i, D}(k)$. A subband regressor $u$ for the subfilters $w_{i}(k)$ of length $M_{s}$, is defined as follows:

$$
\begin{gathered}
u_{i}(k) \equiv\left[u_{i, D}(k), u_{i, D}(k-1), \ldots u_{i, D}\left(k-M_{s}\right.\right. \\
+1)]^{T}
\end{gathered}
$$

for $i=0,1, \ldots, M-1$, where $D=M / 2$ is the decimation factor. Each column of $u$ holds a subband regression vector. The use of FFT to decompose the signals into subbands leads to significant amount of computational savings; however, it introduces circular convolution and circular correlation. This can be further nullified by overlapping of input samples.

Delayless subband system eliminates the signal path delay caused by the analysis and synthesis filter banks. The fullband filtered reference signal and the pseudo error signal is decomposed into number of subbands 
using analysis filters in the DFT filterbanks. All the subband signals are downsampled by the decimation factor $D$. Subband weight adaptation is done by closed loop feedback mechanism. The fullband error signals are fed to subband adaptive filters which finally converges to optimal Weiner solution. The filter weights in each subband is adjusted using complex normalized LMS algorithm defined as:

$$
w_{i}(k+1)=w_{i}(k)+\frac{\mu u_{i}^{*}(k)}{\left\|u_{i}(k)\right\|^{2}} e_{i, D}(k)
$$

Here, $\mu$ is the step size for the adaptation algorithm. Its value affects the convergence speed, steady state error and stability of the adaptive filter. The subbands adaptive weight vector of $m$ th subband are defined as

$$
w_{m}(n)=\left[w_{m_{0}}(n) w_{m_{1}}(n) \ldots w_{m_{P-1}}(n)\right]^{T}
$$

These weights are then transformed from subband to fullband by weight transformation scheme. The filter weights are transformed into frequency domain by $M_{S}$ point FFT; this results in

$W(k)=F F T[W(k)]=\left[W_{0}(k) W_{1}(k) \ldots W_{N-1}(k)\right]^{T}$

where $w_{i}(k)$ is the subband adaptive weights. These weights are properly stacked and then inverse transformed every $N$ samples to get the wide band filter coefficients.

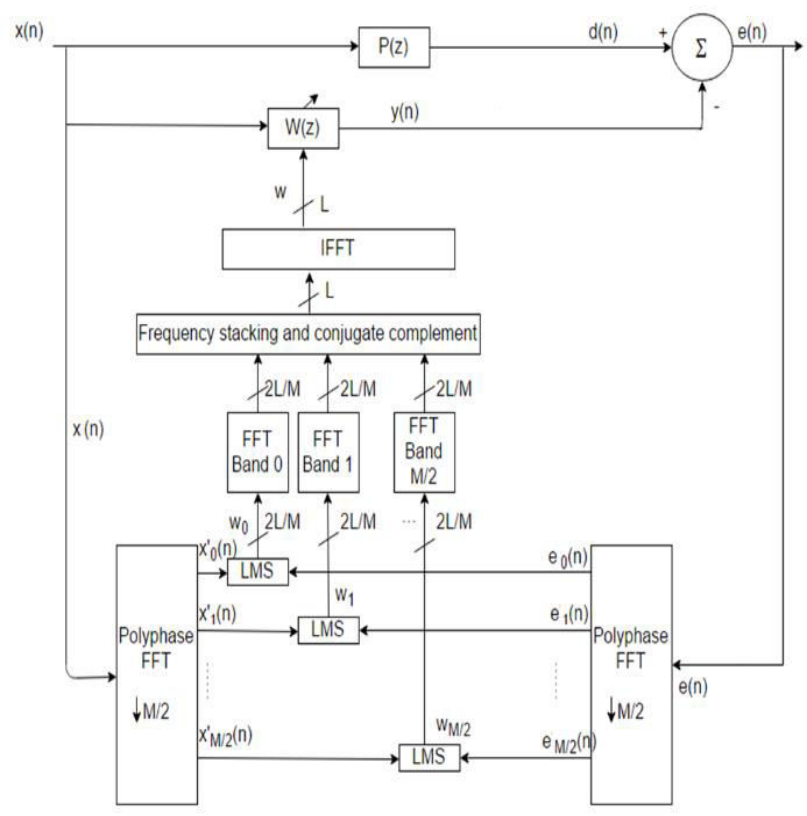

Figure 2. Delayless active sound control using subband.

$$
\begin{aligned}
& w(k)=\operatorname{IFFT}[W(k)] \\
& =[w(k N-1) w(k N-2) \ldots w(k N-N)]^{T}
\end{aligned}
$$

\section{DIFFERENT WEIGHT TRANSFORMATION SCHEMES}

The weight transformation is greatly dependent on the characteristics of the analysis filter bank used for the subband decomposition. Two different weight transformation schemes are explained below.

\section{A. Frequency Sampling Method ([20])}

In this method DFT filterbank consists of complex modulated bandpass filters. As the subband signals are complex valued, subband adaptive weights are also complex valued. Weight transformation maps the complex subband tap weights into an equivalent set of real valued full band tap weights. The weight transformation consists of the following steps:

1. For the first $M / 2+1$ subbands the weight vectors are transformed by the FFT to obtain Ms DFT coefficients for each subband.

2. The DFT coefficients obtained above are stacked to form the first $L / 2$ points of an $L$ element vector from index 0 to $L / 2-1$. It is completed by setting $L / 2$ th point to zero and then using the complex conjugate values from index 1 to $L / 2-1$ in reversed order. The inverse FFT of the $L$ element vector gives the fullband tap weights. The frequency stacking rules are listed as follows:

1. For $l \in\{0 ; L / 2-1\}, W(l)=W_{p}(q)$, where $W(l)$ and $W_{p}(q)$ denote the FFT coefficients of the fullband filter and the $p t h$ subband filter, respectively; $p=[l M / L]$ where [.] denotes the rounding towards the nearest integer; and $q=l_{2 L / M}$, where $a_{b}$ denotes $a$ modulus $b$.

2. For $l / L / 2, W(L / 2)=0$

3. For $l \in\{L / 2+1 ; L-1\}, W(l)=W(L-1)^{*}$.

\section{B. DFT filter bank with fractional delays ([21])}

Weight transformation for critically decimated subband adaptive filtering can be done by using DFT filterbank by using lowpass prototype filter as $M$ th band filter. Analysis DFT filter bank with fractional delays can be obtained by using last polyphase component as $E_{N-1}(z)=z^{-\Delta_{\text {int }}}$ where $\Delta_{\text {int }}$ denotes the integer part of the delay. Also, the length of the adaptive subfilters needs to be increased by one sample for accurate modelling of the unknown system. The subband tap weights to full band weight transformation can be done through the following steps:

1. Compute an $N$ point IFFT on each of $M_{S}$ columns of the matrix formed by impulse response of the adaptive subfilters $W_{i}(z)$. This result gives the impulse response of the fractionally delayed polyphase component $G_{i}^{\prime}(z)=G_{i}(z) z^{i / N}$, where $G_{0}(z), \ldots, G_{N-1}(z)$ are the polyphase component of the fullband filter $W(z)$.

2. Take $G_{0}(z)=G_{0}^{\prime}(z)$, for the first polyphase component. For the consequent components the impulse response of $G_{i}^{\prime}(z)$ is convolved with the fractional delay $E_{i-1}(z)$ as

$$
G_{i}(z) z^{-\left(\Delta_{i n t}+1\right)}=G_{i}^{\prime}(z) E_{i-1}(z)
$$

3. Discard the first polyphase component, discard the last sample and for the subsequent samples discard the first $\Delta_{\text {int }}+1$ samples and retain the next $M_{s}-1$ samples. The fullband filter can be constructed from these polyphase components as

$$
W(z)=\sum_{i=0}^{N-1} G_{i}\left(z^{N}\right) z^{-1}
$$


The computational complexity of the delayless subband structure can be divided into these parts:

1. Filter bank operations

2. Subband weight adaptation

3. Fullband filtering

4. Weight transformation

The detailed breakdown of computational requirements of two weighting transformation schemes can be found in references $[20,21]$ and is not repeated here. The computational complexity for Morgan and Merched algorithms are plotted with varying number of subbands in Fig. 3. As can be seen Merched performs much better from this point of view. Moreover in both algorithms by increase in the number of subbands delayless structure impose less computational burden to the whole algorithm.

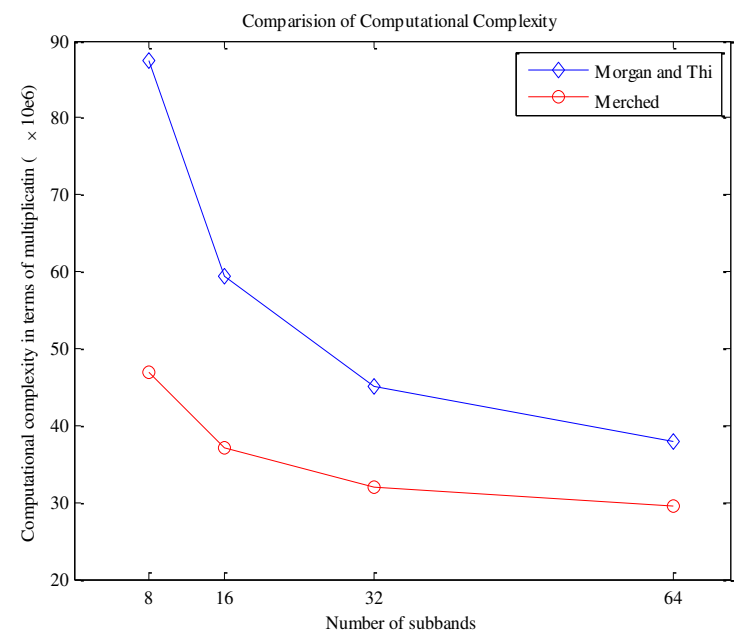

Figure 3. Comparison of computational complexity of the algorithms proposed in [10] and [11].

\section{SIMULATION RESULTS}

Simulations were done in MATLAB to verify the performance of the proposed delayless active noise control algorithm. Two different types of signals were used as the excitation signal to identify the secondary path. Speech signal with sampling frequency of $16 \mathrm{KHz}$ and music signal with sampling frequency of $44.1 \mathrm{KHz}$ were considered for this purpose. The reason for selection of these two signal types is the fact that these are usually the audio signals sent through secondary loudspeakers in an integrated audio and ANC system. This aids to avoid injection of auxiliary annoying noise during the operation of system. Further to that it is worthy to note that these two signals have different frequency and non-stationary characteristics that make adaptive identification of secondary path a challenging task. The energy of the speech signal is concentrated mostly at lower frequencies whereas music has a broader range. In time domain, music signal is fairly continuous but speech signal have paused between words and phrases. For comparison the frequency spectrum of speech and music signals are plotted in Figs. 4 and 5 respectively.

The experimental ANC setup in which the secondary path $S(z)$ is aimed to be identified is shown in Fig. 1. The reference signal $u(n)$ is subjected to polyphase FFT transformation with blocks of 1024 samples for each iteration. The prototype low pass filter used to achieve the DFT filterbank is selected to be of the order 255. It has passband edge frequency at $6 \mathrm{KHz}$ and cutoff frequency of $\pi / 8$. The bandpass filters in the subbands are the frequency shifted version of the prototype low pass filter. The length of the full band adaptive weight vector is 1024 . $M=32$ filter bank is used with each subband having $8 M$ weight vectors. The value of the stepsize $\mu$ has been set differently for different algorithms. The value of stepsize for Merched algorithm is set to be 0.3 , however, this value for Morgan algorithm is 0.2 for the speech as excitation signal. For the music signal it is set to 0.15 . The values of step sizes are chosen such that the fastest possible response is achieved before the adaptive algorithms go unstable.

The minimum mean square error of the identification error after convergence of the adaptive algorithms for both music and speech signals compared to the timedomain NLMS algorithm are plotted in Figs. 6, 7, 8 and 9. As can be seen the attained minimum mean square error for Merched algorithm is better that the two others. Besides among the three algorithms the time domain NLMS algorithm is not converging to the right model at all. To compare the algorithms in terms of the speed of convergence the normalized misalignment is calculated from equation (9).

Normalized misalignment $=20 \log _{10} \frac{\|w(k)-b\|}{\|b\|}$

This value shows the distance of the adaptive weight vector form its optimal weights vector $b$ by calculating their norm. The learning curves for Merched and Morgan algorithms for both speech and music signals are shown in Figs. 10 and 11 by plotting the normalized misalignment given in (9). Comparison of results with respect to the time-domain NLMS algorithm reveals that Merched algorithm performs much better than all other algorithms and time domain NLMS algorithm has the slowest convergence rate. Considering all simulation results it can be seen that Merched algorithm has superior performance with respect to other in term sof computational complexity, convergence speed and achievable minimum mean square error.

\section{CONCLUSION}

Analysis and synthesis filter banks are essential parts of the subband adaptive filtering. However, they tend to increase the overall signal path. In this paper, the performances of two frequency domain delayless adaptive filters for identification of the secondary path in an ANC system are compared. Two types of audio signals, i.e. music and speech signals are used as excitation signals for adaptive system identification. Simulation results show that due to the wideband and non-stationary nature of the excitation signals commonly used time-domain NLMS algorithm fails to give an accurate result. Nevertheless, among subband algorithms Merched is performing significantly better than the others 
both in terms of convergence speed and achievable minimum mean square error. This is due to the fact that the use of fractional delay in the weight transformation scheme will cancel aliasing effects in subbands. It is also proved that this algorithm is less computational complex than the others.

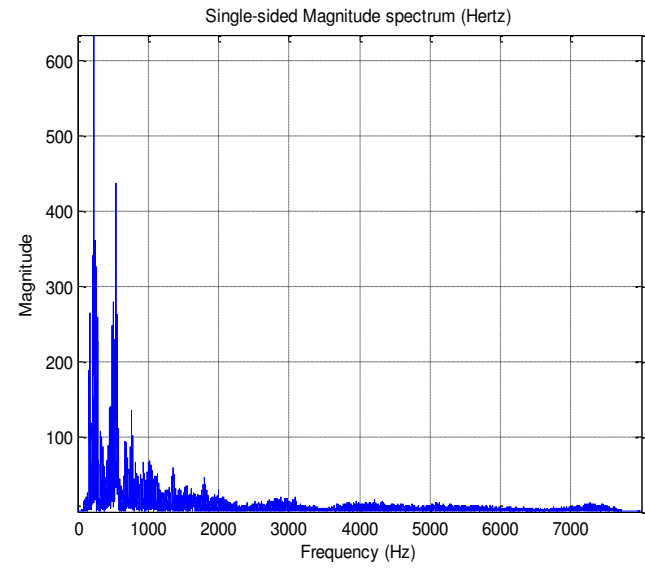

Figure 4. Single sided amplitude spectrum of speech.

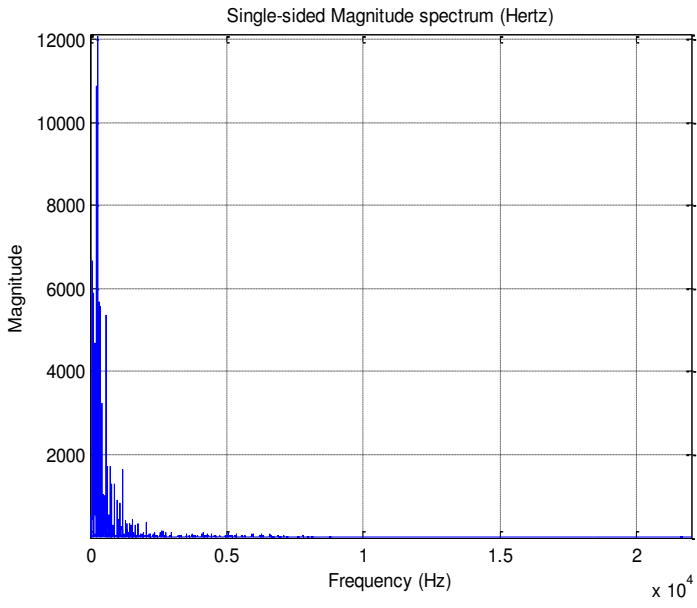

Figure 5. Single sided amplitude spectrum of music.

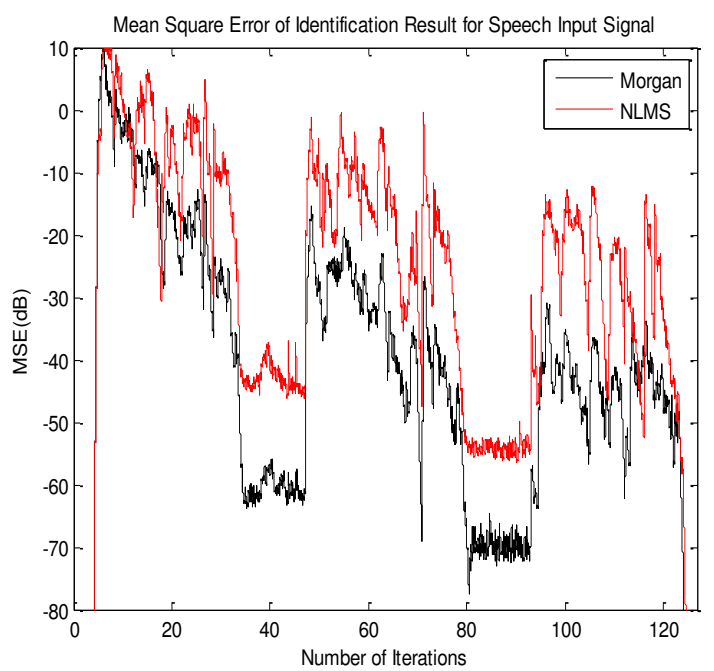

Figure 6. Convergence in terms of Mean Square Error (in dB): Speech (Merched vs NLMS).

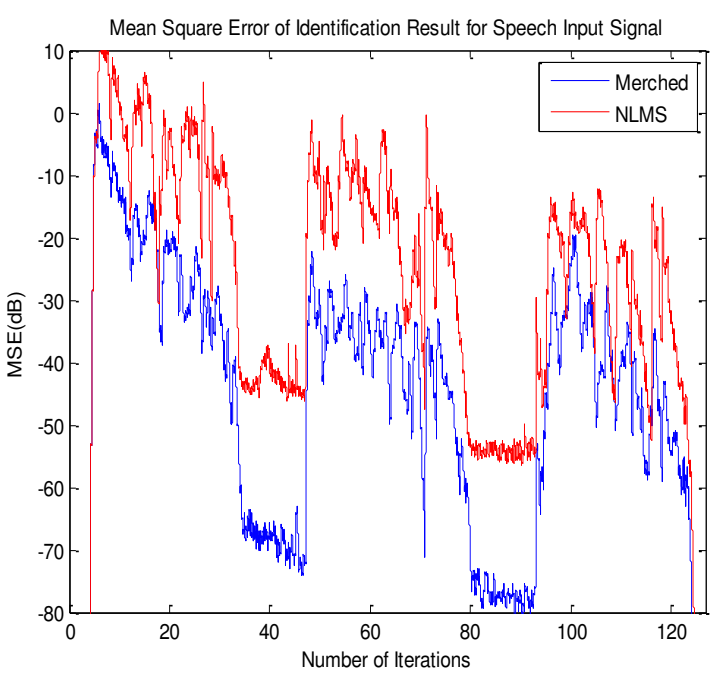

Figure 7. Convergence in terms of Mean Square Error (in dB): Speech (Morgan vs NLMS)

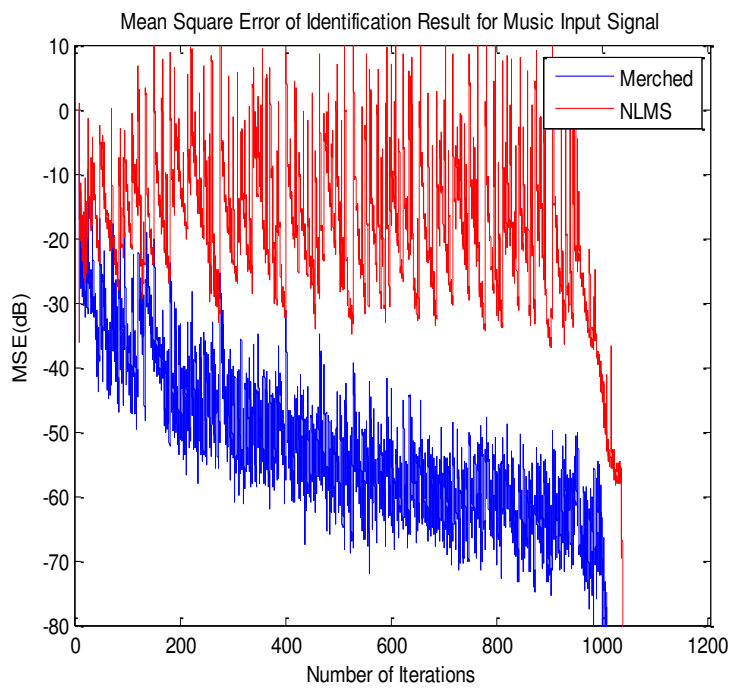

Figure 8. Convergence in terms of Mean Square Error (in dB): Music (Merched vs NLMS).

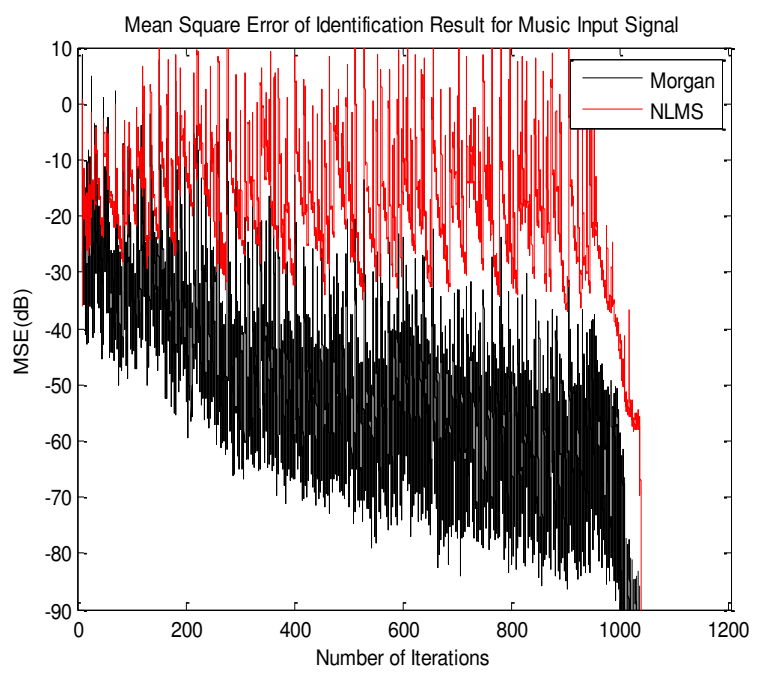

Figure 9. Convergence in terms of Mean Square Error (in dB): Music (Morgan vs NLMS). 


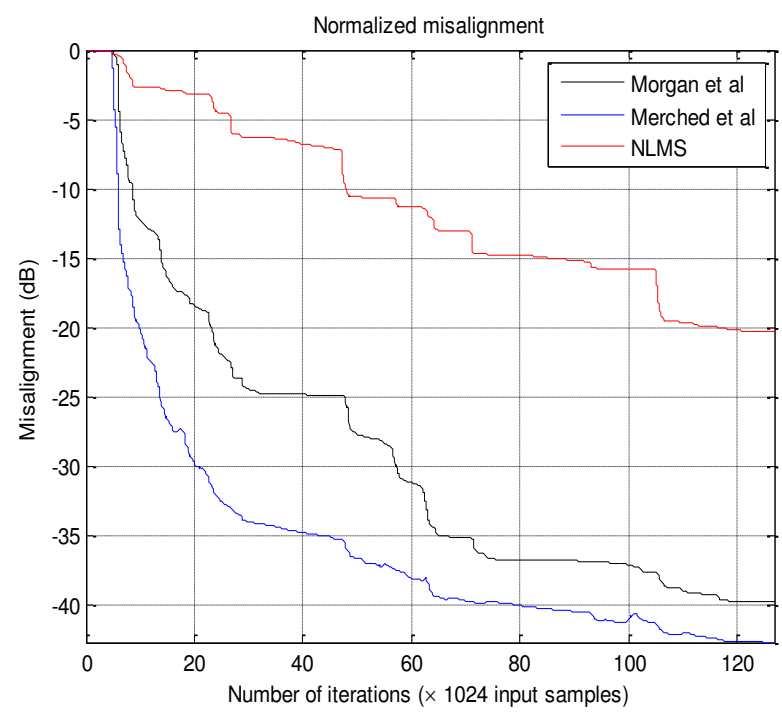

Figure 100. Normalized misalignment learning curves: Speech

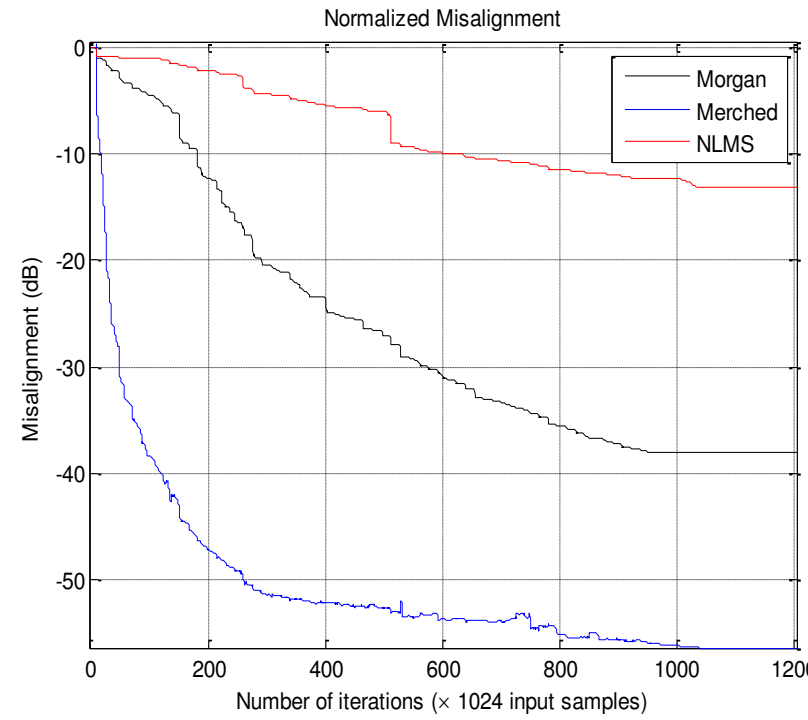

Figure 111. Normalized misalignment learning curves: Music

\section{REFERENCES}

[1] P. A. Nelson and S. J. Elliott, Active control of sound. Academic Press, 1991.

[2] A. Montazeri, Modeling, Optimizing Sensors and Actuators Configuration and Developing Algorithms in Active Noise and Vibration Control Systems. PhD Thesis, Iran. Univ. of Science and Technology, 2009.

[3] C.Alippi, D.Cogliati "Active Noise Control: Compensating Nonstationary Acoustic Signals," IEEE International Conference on Intelligent Measurement Systems and Applications, Italy, July 2005 .

[4] A. Barkefors, M. Sternad, "Adapting an MSE controller for active noise control to nonstationary noise statistics," Internoise 2014, Melborn 2014.

[5] ID Landau, T.B. Airimitoaie, A.C. Silva, "Adaptive attenuation of unknown and time-varying narrow band and broadband disturbances," International Journal of Adaptive Control and Signal Processing, March 2015.

[6] E. Bjarnason, "Analysis of the Filtered-x LMS Algorithm,"
IEEE Transactions on Speech and Audio Processing, vol. 3, No. 6, Nov. 1995.

[7] P.L. Feintuch, N.J. Bershad, A.K. Lo, "A Frequency Domain Model for Filtered LMS algorithms- Stability Analysis, Design, and Elimination of Training Mode," IEEE Transactions on Signal Processing, vol. 41, No. 4, April 1993.

[8] J.K. Thomas, SP Lovstedt, JD. Blotter, SD. Sommerfeldt, "Eigenvalue equalization filtered-x algorithm for the multichannel active noise control of stationary and nonstationary signals," Journal of Acoustic Society of America, vol. 123(6), pp. 4238-49.

[9] A. Montazeri, J. Poshtan, "A Computationally Efficient Adaptive IIR Solution to Active Noise and Vibration Control Systems," IEEE Trans. on Autom. Cont., vol. 55, no. 11, pp. 2671-2676, 2010.

[10] A. Montazeri, J. Poshtan, "A new adaptive recursive RLS-based fast array IIR filter for active noise and vibration control systems," Signal Processing, vol. 91, no. 1, pp. 98-113. 2011.

[11] A. Montazeri, J. Poshtan, "Design and analysis of an RLS-Type modified filtered-x algorithm for adaptive IIR filters," Proc. of IEEE International Conference on Signal Processing, pp. 219-222, Beijing, China, 2008.

[12] S.-C. Chan, Y. Chu, "Performance Analysis and Design of FxLMS Algorithm in Broadband ANC System with Online SecondaryPath Modeling," IEEE Transactions on Audio, Speech, and Language Processing, vol. 20, no. 3, pp. 982 - 9932011.

[13] M. Zhang, H. Lan, W. Ser, "Cross-updated active noise control system with online secondary path modeling," IEEE Trans. on Speech and Audio Processing, vol. 9, no. 5, pp. 598-602.

[14] P. Davari, H. Hassanpour, "A variable step-size FxLMS algorithm for feedforward active noise control systems based on a new online secondary path modelling technique," IEEE/ACS International Conference on Computer Systems and Applications, pp. 74-81, March 2008. .

[15] A. Gilloire, "Experiments with sub-band acoustic echo cancellers for teleconferencing," IEEE International Conference on Acoustics, Speech and Signal Processing, vol. 12, pp. 2141-2144, 1987.

[16] H. Yasukawa, S. Shimada, and I. Furukawa, "Acoustic echo canceller with high speech quality," IEEE International Conference on Acoustics, Speech and Signal Processing, vol. 12, pp. 2125-2128, 1987.

[17] S. Sandeep Pradham and V. U. Reddy, "A new approach to subband adaptive filtering," IEEE Transactions on Signal Processing, vol. 47, no. 3, pp. 655-664, Mar. 1999.

[18] M. R. Petraglia, R. G. Alves, and P. S. R. Diniz, "New structures for adaptive filtering in subbands with critical sampling," IEEE Transactions on Signal Processing, vol. 48, no. 12, pp. 33163327, Dec. 2000.

[19] A. Gilloire and M. Vetterli, "Adaptive filtering in subbands with critical sampling: analysis, experiments, and application to acoustic echo cancellation," IEEE Transactions on Signal Processing, vol. 40, no. 8, pp. 1862-1875, 1992.

[20] D. R. Morgan and J. C. Thi, "A delayless subband adaptive filter architecture," IEEE Transactions on Signal Processing, vol. 43, no. 8, pp. 1819-1830, Aug. 1995.

[21] R. Merched, P. S. Diniz, and M. R. Petraglia, "A new delayless subband adaptive filter structure," IEEE Transactions on Signal Processing, vol. 47, no. 6, pp. 1580-1591, 1999.

[22] K.-A. Lee, W.-S. Gan, and S. M. Kuo, Subband Adaptive Filtering: Theory and Implementation. John Wiley \& Sons, 2009.

[23] A. Montazeri, J. Reger, "Developing an IIR Robust Adaptive Algorithm in the Modified Filtered-x RLS Form for Active Noise and Vibration Control Systems," 50th IEEE Conference on Decision and Control and European Control Conference, IEEE CDC 2011, Orlando, USA, 2011. 